2018]. Disponible en: http://temas.sld.cu/dengue/2017/12/27/ manual-washington-de-especialidades-clinicas-enfermedadesinfecciosas/

5. Alves A, Moura D, Spolti G. Co-infection with hepatitis A and leptospirosis in the Amazon region: report of two cases. Trop Gastroenterol. 2011;32(3):234-6.

6. Núñez-Garbín A, Espinoza-Figueroa J, Sihuincha-Maldonado M, Suarez-Ognio L. Coinfección por dengue y leptospirosis en una niña de la Amazonía peruana. Rev Peru Med Exp Salud Publica. 2015 ;32(1):179-82.

7. Jaramillo L, Arboleda M, García V, Agudelo-Flórez P. Coinfección brucelosis-leptospirosis, Urabá, Colombia. Reporte de caso. Infectio. 2018;18(2):72-6.

Correspondencia: Jorge Luis Fernández Mogollón

Dirección: Jr. San José 143 - Lambayeque, Perú.

Correo electrónico:jorgeluis_59@yahoo.es

\section{INFECCIÓN POR VIRUS LINFOTRÓPICO DE CÉLULAS T HUMANAS TIPO 1 EN PACIENTES CON DIAGNÓSTICO DE TUBERCULOSIS MULTIDROGORRESISTENTE}

\section{INFECTION BY HUMAN T-CELL LYMPHOTROPIC VIRUS TYPE 1 IN PATIENTS DIAGNOSED WITH MULTIDRUG-RESISTANT TUBERCULOSIS}

\author{
Rodrigo Cachay ${ }^{1,3, a}$, Alvaro Schwalb ${ }^{1,3, a}$, \\ Romina Mora ${ }^{1,2, b}$, Tatiana Cáceres ${ }^{1,3, b, c}$, Eduardo Gotuzzo ${ }^{1, d}$
}

Perú es uno de los países de las Américas que tiene una alta incidencia de tuberculosis (TB) ${ }^{(1)}$; además, se estima que tiene una prevalencia de $2,5 \%$ de serología positiva

\footnotetext{
1 Instituto de Medicina Tropical "Alexander von Humboldt", Universidad Peruana Cayetano Heredia, Lima, Perú

2 Unidad de Virología, Unidad de Epidemiología Molecular, Universidad Peruana Cayetano Heredia, Lima, Perú

3 Unidad de Investigación de Tuberculosis, Universidad Peruana Cayetano Heredia, Lima, Perú

a Médico cirujano; ${ }^{\mathrm{b}} \mathrm{BsC} ;{ }^{\mathrm{c}} \mathrm{MPhH} ;{ }^{\mathrm{d}}$ médico, especialista en Enfermedades Infecciosas y Tropicales

Recibido: 25/01/2019 Aprobado: 06/03/2019 En línea: 15/03/2019
}

Citar como: Cachay R, Schwalb A, Mora R, Cáceres T, Gotuzzo E. Infección por virus linfotrópico de células $\mathrm{T}$ humanas tipo 1 en pacientes con diagnóstico de tuberculosis multidrogorresistente. Rev Peru Med Exp Salud Publica. 2019;36(1):150-1.doi:10.17843/rpmesp.2019.361.4232. para el virus linfotrópico de células $\mathrm{T}$ humanas tipo 1 (HTLV-1) ${ }^{(2)}$. Esta prevalencia se incrementa hasta $5,8 \%$ en pacientes con TB, según un estudio realizado en el distrito de San Juan de Lurigancho (SJL) en Lima (1). El riesgo de desarrollar TB en pacientes portadores del virus es de dos a cuatro veces mayor que en las personas no infectadas ${ }^{(3)}$.

Se ha demostrado que la infección por HTLV-1 disminuye la respuesta inmune tipo 1 al antígeno micobacteriano, por lo que la presencia del virus en pacientes con TB supone un curso de enfermedad más complicado ${ }^{(3)}$. Por ejemplo, en los casos de coinfección con HTLV-1, se evidencia 3,5 veces más lesiones de destrucción parenquimal que en los casos de TB pulmonar ${ }^{(3)}$. Por otro lado, la presencia de TB induce una respuesta inflamatoria exagerada que contribuye al desarrollo de la mielopatía asociada a HTLV-1 (4). Además, debido a que estos pacientes presentan esputo con mayor carga bacilar por mayor tiempo (1), el pobre control contra el bacilo podría resultar en el desarrollo de cepas de tuberculosis multidrogorresistente (TB-MDR). Considerando el daño resultante a nivel pulmonar, así como su considerable incremento en la infectividad, la asociación entre ambos patógenos amerita estudios más amplios.

En nuestro estudio, se analizó retrospectivamente la serología para HTLV-1 en 106 muestras de pacientes provenientes del distrito de SJL, con diagnóstico de TBMDR (resistente a isoniacida y rifampicina). Las muestras provenían de estudios previos realizados por la Universidad Peruana Cayetano Heredia, los cuales estaban destinados a la formación de un banco de muestras para el desarrollo de pruebas diagnósticas y hallazgos de marcadores asociados a TB. Encontramos que 2,8 \% (IC 95\%, 0,72-7,5) tuvieron serología positiva para el virus. No hemos encontrados estudios a nivel nacional que midan cuantitativamente la proporción de portadores de HTLV-1 en pacientes con TB-MDR. Esta asociación podría ser un factor que favorece la alta prevalencia de TB en el distrito ${ }^{(5)}$.

En la muestra, también se analizó la frecuencia de infección por el virus de la inmunodeficiencia humana $(\mathrm{VIH})$, la cual fue de 4,7\% (IC 95\%: 2,0-10,5); no se detectó ningún caso de coinfección por ambos retrovirus. Según Verdonck et al, la tasa de infección dual (VIH y HTLV-1) es 3,7\% en pacientes hospitalizados por TB. Se ha estudiado previamente el impacto que ha tenido el VIH en el incremento de la proporción de personas que desarrollan TB activa ${ }^{(6)}$; por lo que también se le considera un mediador importante de la aparición de numerosos casos de TB-MDR.

Debido que se trabajó solamente con información laboratorial, no se puede determinar una asociación más precisa entre los factores clínicos y epidemiológicos. Además, debido a la falta de presupuesto no se contó con sueros y reactivos suficientes, lo que limitó considerablemente el tamaño de muestra. 
Si bien, en la literatura se ha reportado una prevalencia de infección por HTLV-1 de 5,8\% de pacientes con TB en el mismo distrito, no se especifica el patrón de resistencia de estos casos. Por lo tanto, los resultados de nuestro estudio podrían sugerir que la proporción de HTLV-1 en pacientes con TB-MDR es elevada. Por esta razón, consideramos importante dirigir el enfoque clínico para aminorar el compromiso pulmonar $\mathrm{y} / \mathrm{o}$ atender oportunamente a las posibles complicaciones. Asimismo, dada su mayor infectividad, es necesario la aplicación de estrategias para disminuir su impacto en la salud pública. Se necesitan estudios de mayor tamaño muestral para conocer con mayor exactitud esta proporción y así evaluar la necesidad de un tamizaje de HTLV-1 en pacientes del Programa Nacional de Control y Prevención de Tuberculosis.

Contribuciones de autoría: Todos los autores participaron en la concepción y diseño del artículo. RM y TC participaron en la recolección de datos. RC y AS participaron en la redacción del artículo, EG participó en la revisión crítica del artículo y aprobación de la versión final.

Financiamiento: Los autores no declaran financiamiento

Conflicto de interés: Los autores declaran no tener conflictos de interés en la publicación de este artículo.

\section{REFERENCIAS BIBLIOGRÁFICAS}

1. Verdonck K, González E, Henostroza G, Nabeta P, Llanos F, Cornejo $\mathrm{H}$, et al. HTLV-1 infection is frequent among outpatients with pulmonary tuberculosis in northern Lima, Peru. Int J Tuberc Lung Dis. 2007;11(10):1066-72.

2. Sanchez-Palacios C, Gotuzzo E, Vandamme A-M, Maldonado Y. Seroprevalence and risk factors for human T-cell lymphotropic virus (HTLV-I) infection among ethnically and geographically diverse Peruvian women. Int J Infect Dis. 2003;7(2):132-7.

3. deLourdes Bastos M, Santos SB. Influence of HTLV-1 on the clinical, microbiologic and immunologic presentation of tuberculosis. BMC Infect Dis. 2012; doi: 10.1186/1471-2334-199.

4. de Lourdes Bastos M, Neves Y, Carvalho N, Lisboa N, Souza A, Santos S, et al. Clinic and immunologic features of the coinfection tuberculosis and HTLV-1. Eur Respir J. 2014; 44 (Suppl 58): P2634.

5. Shah L, Choi HW, Berrang-Ford L, Henostroza G, Krapp F, Zamudio $\mathrm{C}$, et al. Geographic predictors of primary multidrugresistant tuberculosis cases in an endemic area of Lima, Peru. Int J Tuberc Lung Dis. 2014 Nov; 18 (11): 1307-14. doi: 10.5588/ ijtld.14.0011.

6. Verdonck K, Henriquez C, Echevarria J, Huayanay L, Agapito $\mathrm{J}$, Cairampona $\mathrm{R}$, et al. Asociación entre infección por el virus linfotrópico humano de células T tipo I (HTLV-I) y mortalidad en pacientes hospitalizados con tuberculosis. Rev Méd Hered. 2004; 15(4): 197-202. doi: 10.20453/rmh.v15i4.773.

Correspondencia: Rodrigo Cachay

Dirección: Av. Honorio Delgado, 430, Urb Ingenieria, Lima, Perú

Correo electrónico: rodrigo.cachay.f@upch.pe 